\title{
Spontaneous Rupture of Infected Walled-Off Necrosis in the Transverse Colon with a Nonoperative Favorable Outcome: A Rare Event
}

\author{
Virender Chauhan ${ }^{1}$ Gaurav Kumar Gupta ${ }^{1} \quad$ Vasudha Goel $^{1} \quad$ Dilip Singh Mudgal $^{1} \quad$ Mukesh Jain ${ }^{1}$ \\ Sandeep Nijhawan ${ }^{1}$
}

${ }^{1}$ Department of Gastroenterology, SMS Hospital, Jaipur, Rajasthan,

Address for correspondence Gaurav Kumar Gupta, MD, DM, India Department of Gastroenterology, SMS Hospital, Jawahar Lal Nehru Marg, Jaipur 302004, Rajasthan, India (e-mail: kumarggauravpgi@gmail.com).

J Digest Endosc 2020;10:237-239

\begin{abstract}
Infected walled-off necrosis (WON) is a well-known complication of acute necrotizing pancreatitis, with higher mortality and morbidity. An infected or symptomatic WON

Keywords

- walled-off necrosis

- spontaneous rupture

- colon requires drainage. Occasionally, WON may spontaneously fistulize into the gastrointestinal lumen or may rupture into the peritoneum. We describe a case of spontaneous rupture of WON in the transverse colon with uncomplicated spontaneous resolution, which is an extremely rare event.
\end{abstract}

\section{Introduction}

In the revised 2012 Atlanta guidelines of acute pancreatitis, walled-off necrosis (WON) is defined as an encapsulated collection of pancreatic or peripancreatic necrosis that has a well-defined wall and forms at 4 weeks or later after the onset of acute pancreatitis. It can be either sterile or infected. ${ }^{1}$ Spontaneous rupture of WON in the gastrointestinal lumen is a rare event, and only a few case reports have described it. ${ }^{2-}$ ${ }^{4}$ Ever since the introduction of this new terminology, there is no reported case of spontaneous fistulization of infected WON in the colon. We report a case of spontaneous rupture of WON in the transverse colon.

\section{Case Report}

A 50-year-old male patient presented with a history of recurrent vomiting, intermittent fever, loss of appetite, and generalized weakness for 15 days. He had an episode of acute severe necrotizing biliary pancreatitis 8 weeks ago with development of acute kidney injury for which he underwent two sessions of hemodialysis. He developed acute necrotic collection and was managed conservatively. Contrast-enhanced computed tomography (CECT) performed after the first week of acute pancreatitis showed a $7.6 \times 13 \mathrm{~cm}$ heterogeneous ill-defined collection replacing pancreatic parenchyma suggestive of acute fluid collection ( $\boldsymbol{- \text { Fig. }} \mathbf{1}$ ). There was an inflammatory thickening of the wall of the stomach, with multiple fluid collections in peripancreatic, gastrosplenic, and pararenal spaces. Moderate ascites was present. The CT scan also showed multiple small calculi in the gallbladder.

On presentation, he had a pulse rate of $98 /$ minute and blood pressure of 110/74 mm Hg. Abdominal examination

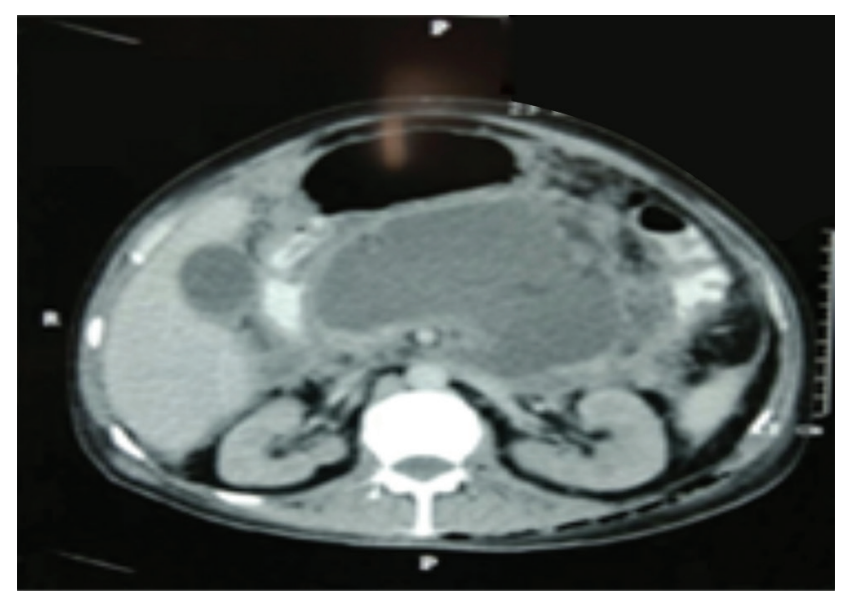

Fig. 1 Pancreatic acute fluid collection before spontaneous rupture. 
revealed a vague tender lump in the epigastric region. His hemoglobin was $6.2 \mathrm{~g} / \mathrm{dL}$, total leucocyte count (TLC) was $19,000 / \mathrm{cu} / \mathrm{mm}$, platelets was $0.52 \mathrm{lacs} / \mathrm{mL}$, SGOT/SGPT (aspartate aminotransferase/alanine aminotransferase) was 19/15 U/L, serum alkaline phosphatase was100 IU/L, serum albumin was $1.8 \mathrm{~g} / \mathrm{dL}$, and serum creatinine was $0.9 \mathrm{mg} / \mathrm{dL}$. The patient was managed with intravenous meropenem and fluconazole, and 1 unit of blood transfusion was performed. On the fifth day of admission, patient developed a single episode of hematochezia, with a fall in hemoglobin of $2 \mathrm{~g} / \mathrm{dL}$. The patient was stabilized with intravenous fluids and 1 unit of blood transfusion. Gastroscopy was normal. Colonoscopy was performed the next morning, which revealed a large ulcerated area in the transverse colon with multiple fistulous openings suggestive of spontaneous rupture of WON (-Fig. 2A, B). Fortunately, bleeding stopped spontaneously. CECT of the abdomen showed small pancreatic collection along with contrast extravasation from the midtransverse colon extending to the body and tail of the pancreas suggestive of pancreatic colonic fistula ( - Fig. 2 ). The patient had a marked relief in vomiting, and his epigastric lump decreased in size. The patient became afebrile, and all his symptoms subsided slowly. His hemoglobin remained stable at $6.8 \mathrm{~g} / \mathrm{dL}$, TLC was $12,000 / \mathrm{cu} / \mathrm{mm}$, and platelet count was $1.1 \mathrm{lacs} / \mathrm{mL}$, indicating improvement. The patient was managed conservatively and kept on strict follow-up after discharge. After 4 weeks, a repeat colonoscopy revealed significant healing of the fistula ( $\mathbf{- F i g}$. 3C), and his general condition improved. Colonoscopy at 8 weeks showed normal colon without any communication. Then the patient was referred for cholecystectomy.

\section{Discussion}

Infected WON is a serious complication that may ensue at least 4 weeks after an episode of acute pancreatitis, with a mortality rate of 20 to $30 \% .^{5}$ According to the 2012 Atlanta guidelines, the various well-defined local complications of acute pancreatitis include acute peripancreatic fluid collection, pseudocyst, acute necrotic collection, and WON. ${ }^{1}$
Conservative management is usually advocated in asymptomatic WON, whereas drainage is required in cases of infected WON or when there is refractory abdominal pain, failure to thrive, and gastric and biliary obstruction.

In acute pancreatitis complications, colonic involvement has a relatively low incidence $(\sim 3 \%) .{ }^{6}$ Colonic complications range from a localized pseudo-obstruction of the ileus to necrosis, hemorrhage, ischemic colitis, and fistula formation. There are many case reports and series describing spontaneous rupture or fistulization of pseudocysts into the adjacent structures such as the stomach, duodenum, or colon, resulting in either further complications or resolution of the condition. ${ }^{7-9}$ In a study, Doberneck had reported closure of fistulas with conservative management in three-fourths of duodenal fistula and one-third of jejunal fistula, whereas all five colonic fistulas in his series required surgery. ${ }^{10}$ There is scarce literature on spontaneous rupture of WON, with a few case reports showing rupture in the stomach and duodenum, with uncomplicated resolution..$^{2-4}$ In our case, the spontaneous rupture of infected WON in the transverse colon with nonoperative favorable outcomes is an extremely rare event and, to the best of our knowledge, has never been reported earlier.

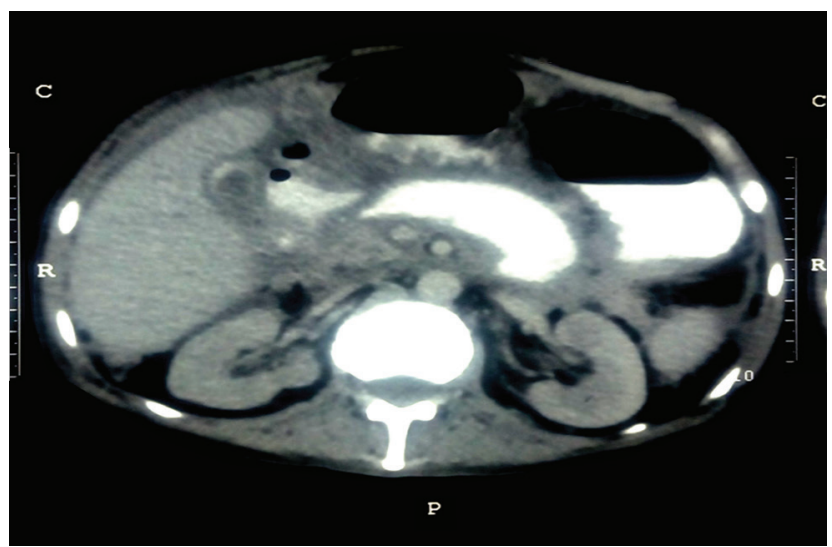

Fig. 3 Walled-off necrosis after spontaneous rupture, with decrease in the size of collection and extravasation of contrast from the colonic lumen into the pancreatic collection.
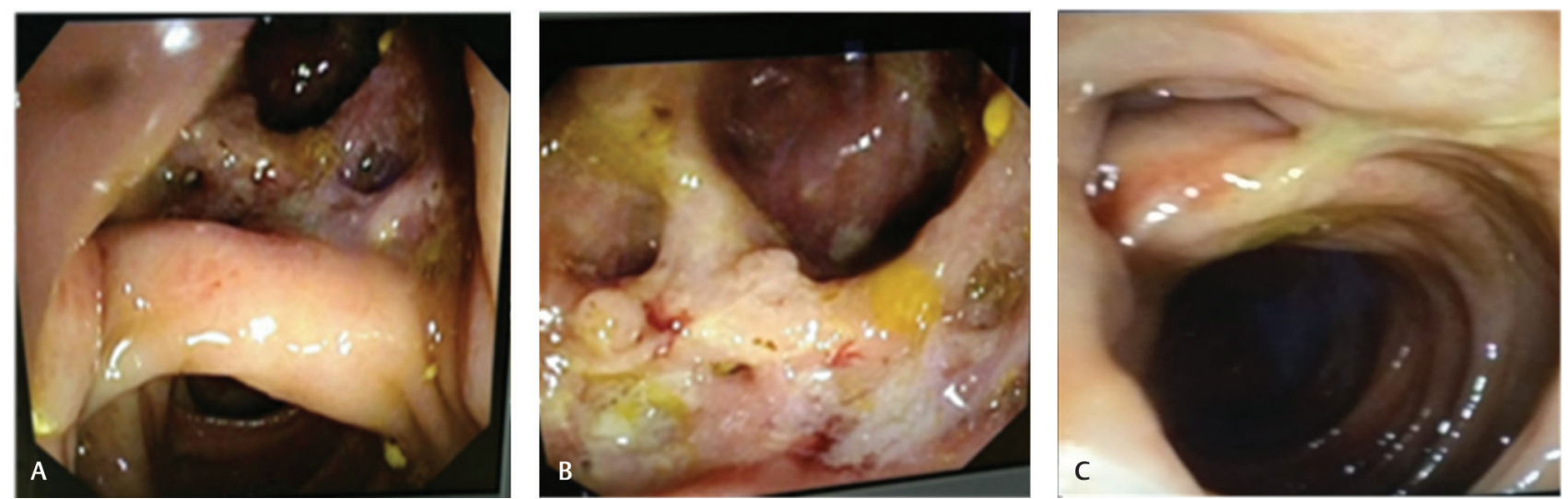

Fig. 2 Colonoscopic view of the transverse colon with multiple fistulous opening from ruptured walled-off necrosis (A, B) before and (C) after spontaneous healing. 


\section{Conflict of Interest}

None.

\section{References}

1 Banks PA, Bollen TL, Dervenis C, et al. Acute Pancreatitis Classification Working Group. Classification of acute pancreatitis-2012: revision of the Atlanta classification and definitions by international consensus. Gut 2013;62(1):102-111

2 Papadimitriou N, Robotis J, Rokkas T. Spontaneous intragastric walled-off pancreatic necrosis rupture. JOP 2014;15 (6):628-629

3 Boopathy V, Balasubramanian P, Alexander T, Koshy R. Spontaneous fistulisation of infected walled-off necrosis (WON) into the duodenum in a patient following acute necrotising pancreatitis. BMJ Case Rep 2014;2014:202863

4 Ran SS, Sharma V, Prasad KR, et al. Spontaneous intra-gastric rupture of walled-off pancreatic necrosis: endoscopic ultrasound features. J Dig Endosc 2016;7:160-162
5 Werge M, Novovic S, Schmidt PN, Gluud LL. Infection increases mortality in necrotizing pancreatitis: A systematic review and meta-analysis. Pancreatology 2016;16(5):698-707

6 Mohamed SR, Siriwardena AK. Understanding the colonic complications of pancreatitis. Pancreatology 2008;8(2):153-158

7 Clements JL, Jr. Bradley EL, III. Eaton SB Jr. Spontaneous internal drainage of pancreatic pseudocysts. AJR Am J Roentgenol 1976;126(5):985-991

8 Uiterwaal MT, Overbosch EH, Bruno MJ, van der Hulst RW. Spontaneous drainage of a pancreatic pseudocyst after embolization of a bleeding pseudoaneurysm. Cardiovasc Intervent Radiol 2009;32(1):192-194

9 Mir MF, Shaheen F, Gojwari TA, Singh M, Nazir P, Ahmad S. Uncomplicated spontaneous rupture of the pancreatic pseudocyst into the gut-CT documentation: a series of two cases. Saudi J Gastroenterol 2009;15(2):135-136

10 Doberneck RC. Intestinal fistula complicating necrotizing pancreatitis. Am J Surg 1989;158(6):581-583, discussion 583-584 